LC-DET-2001-056

February 16, 2001

\title{
TESLA Detector Magnet Design
}

\author{
François Kircher*, Bernard Gastineau*, Viatcheslav Klioukhine*Ill, and Yves \\ Pabot** \\ * DSMDAPNIA/STCM, Bât 123, CEA Saclay, 91191 Gif-Sur-Yvette, France \\ I Now at Fermi National Accelerator Laboratory, Batovia, IL, 60510-0500, USA \\ ** DSMDAPNIA/SGPI, Bât 123, CEA Saclay, 91191 Gif-Sur-Yvette, France
}

\begin{abstract}
The TESLA detector asks for a strong and very homogeneous magnetic field within its useful volume. In this respect, a large superconducting magnet has been designed, with special attention to get the requested field homogeneity. The magnet design is described in this paper, as well as a short description of the main technical choices. Finally, some elements about the planning and cost estimate are given.
\end{abstract}




\section{SUMMARY}

1 Introduction

2 Physics requirements

3 Magnet design

3.1 Generalities

3.2 Main parameters

3.3 Coil characteristics

3.4 Yoke

3.5 Magnetic field homogeneity

4 Technical aspects

4.1 Conductor

4.2 Winding

4.3 Coil mechanical structure

4.4 Cooling

4.5 Yoke

4.6 Assembly

4.7 Field mapping

5 Ancillaries

5.1 Cryogenic plant

5.2 Power circuit

5.3 Control and monitoring system

6 Estimates

6.1 Costs

6.2 Manpower requirements

6.3 Planning

7 Conclusions

References

Figures 


\section{1 - INTRODUCTION}

The basic layout of the TESLA detector has always followed the strategy of tracking in a magnetic field. During the first phase of the study, two versions were compared :

- small detector version : higher field $(5-6 \mathrm{~T})$ in a smaller volume ( $2 \mathrm{~m}$ in diameter) ;

- large detector version : lower field $(3-4 \mathrm{~T})$ in a larger volume (6 $\mathrm{m}$ in diameter).

The large detector version was found to have a better overall performances than the smaller one. So the TESLA detector design asks for a $4 \mathrm{~T}$ field, in a large volume, and with a high field homogeneity within the TPC volume.

As the characteristics of the TESLA magnet are very similar to the CMS one [1], not surprisingly both magnets have a rather similar design [2]. However, an extra challenging point for the TESLA magnet is its required field homogeneity. An original set up of correction coils included in the main winding has been proposed to fulfill this requirement.

\section{2 - PHYSICS REQUIREMENTS}

The main requests for the TESLA Magnet are a solenoidal central field of 4T, in a volume of $6 \mathrm{~m}$ in diameter and $8.5 \mathrm{~m}$ long, and with a high field homogeneity in the TPC volume, a cylinder $3.2 \mathrm{~m}$ in diameter and $5 \mathrm{~m}$ long :

$$
\left|\int_{0}^{2.5} m\left(B_{r} / B_{z}\right) d z\right| \leq 2 \mathrm{~mm}
$$

with

$$
B_{r}=B_{x}(x / r)+B_{y}(y / r)
$$

and $\mathrm{z}$ being the direction of the beam axis.

The field volume mentioned above will be used for inner detectors and consequently must be free from any other material.

The iron of the flux return will be used as an absorber for muon identification and must be segmented in slabs for this purpose. 


\section{3 - MAGNET DESIGN}

\subsection{Generalities}

Except for the field homogeneity request, the TESLA magnet parameters are very similar to the CMS ones ; so both magnets will have a lot of similarities.

The magnet consists of the superconducting coil, a solenoid made of 5 modules and which included correction coils, and of the iron yoke, composed of the central barrel yoke and two end cap yokes.

Preliminary calculations showed that a total coil length of $9.25 \mathrm{~m}$ and an iron thickness of $1.6 \mathrm{~m}$ were good compromises to obtain the requested field parameters.

Concerning the field homogeneity, it appeared quickly that special devices were requested to get it. First option looked at was to add extra correction layers in the winding, such as in the Aleph solenoid case. Due to their dimensions, such devices would have been very complicated to integrate both from the winding and the cryostat point of views.

So, it has seemed practically simpler to incorporate them in the main winding, by adding an extra current in appropriate locations of the winding. A shaping of the end cap yoke was also studied for extra improvement of the field homogeneity.

\subsection{Main parameters}

The cross section of a quadrant of the CMS detector magnet is shown on Fig 1. Its main parameters are given in Tab I, II and III for respectively the geometrical, the magnetic and electrical and the weight parameters.

Tab I : TESLA magnet geometrical parameters

Cryostat inner radius (m)

Coil inner radius (m)

Coil outer radius $(\mathrm{m})$

Cryostat outer radius (m)

Barrel yoke inner radius (m)

4.45

Barrel yoke outer radius (m)

6.45

Coil length (m)

9.25

Cryostat length (m)

10.20

Yoke overall length (m)

14.80 
Tab II : TESLA magnet electrical and magnetic parameters

$\begin{array}{ll}\text { Central field (T) } & 4.00\end{array}$

Maximum field on conductor (T) $\quad 4.70$

Stored energy (GJ) $\quad 2.32$

Main current (A) 18800

Correction current (A) 24500

Ampere turns main coil (A.t) $29.810^{6}$

Ampere turns correction coit (At) $4.710^{6}$

Tab III : TESLA magnet weight

Cold mass (t)

$\begin{array}{ll}\text { Barrel yoke (t) } & 6400\end{array}$

$\begin{array}{ll}\text { End cap yokes }(\mathrm{t}) & 2000\end{array}$

$\begin{array}{ll}\text { Total weight }(\mathrm{t}) & 8600\end{array}$

\subsection{Coil characteristics}

The coil is divided into five modules, electrically and mechanically connected : the three central modules are $2.35 \mathrm{~m}$ long each, with 100 turns per layer and 4 layers ; the two external modules are $1.1 \mathrm{~m}$ long each, with 48 turns per layer and 4 layers also.

The main current of $18.8 \mathrm{kA}$ for a central field of $4 \mathrm{~T}$ runs through all the turns of the solenoid. An extra correction current of $24.5 \mathrm{kA}$ is added in each turn of the two middle layers of the two external modules (i.e. the current is $43.3 \mathrm{kA}$ in each of these turns).

\subsection{Yoke}

The yoke was designed in close relation with the muon detector.

Main chosen options were :

- octagonal shape for both the barrel and end cap yokes ;

- similar splitting of the iron for both yokes; from the inside of the yoke :

. 10 times (10 $\mathrm{cm}$ thick iron slab $+4 \mathrm{~cm}$ air gap for the muon chambers) ; $.60 \mathrm{~cm}$ thick iron to make up the total thickness .

- long barrel yoke option, i.e the barrel yoke is overlapping the end cap yokes. 
Practically, the barrel yoke will be longitudinally split into three parts, 3.9, 7.0 and $3.9 \mathrm{~m}$ long respectively.

A magnetic pole tip will be added inside the end cap yoke to improve the field homogeneity. This pole tip is also split to allow the insert of detectors.

\subsection{Magnetic field homogeneity}

As previously mentioned, the requested field homogeneity is obtained through :

- the correction current added to the main current in the two middle layers of the two external modules ;

- the iron pole tip added to the end cap yoke.

The integral field homogeneity given in (1) has been calculated for the theoretical design of the magnetic structure, including the iron splitting for detector insertion and some assembly gaps within the iron structure. Its maximum value for the nominal parameters is reached at $\mathrm{R}=1.12 \mathrm{~m}$ and is shown on Fig 2 .

The result is better than the requirement, the maximum calculated deviation being $25 \%$ smaller than the allocated one (1.45 $\mathrm{mm}$ vs $2 \mathrm{~mm})$.

For this calculation, it has been checked that the stray-field of the quadrupole doublet close to the interaction point has no influence on the field homogeneity of the detector magnet in the TPC volume.

\section{4 - TECHNICAL ASPECTS}

\subsection{Conductor}

The conductor design is similar to that for CMS [3].

Its consists of a superconducting cable coextruded in a very pure aluminium and mechanically reinforced. Two different conductors are necessary, one carrying the main current, one carrying the main and the extra correction currents.

Practically the increase of the current capacity will be done by increasing the number of the diameter of the strands of the cable, but the overall dimensions of both conductors will be the same (Fig 3).

Both conductors will have a nominal current $\left(\mathrm{I}_{\text {nom }}\right)$ about one third of the critical current for the maximum field they are in $\left(\mathrm{I}_{\text {crit }}\right)$ : 
- conductor with a smaller cable :

$$
\begin{aligned}
& . \mathrm{I}_{\text {nom }}=18.8 \mathrm{kA} \text { with } \mathrm{B}_{\max }=4.7 \mathrm{~T}, \mathrm{~T} \sim 4.5 \mathrm{~K} \\
& . \mathrm{I}_{\text {crit }}=58 \mathrm{kA} \text { for } \mathrm{B}_{\max }=5 \mathrm{~T}, \mathrm{~T}=4.2 \mathrm{~K}
\end{aligned}
$$

- conductor with a larger cable :

$$
\begin{aligned}
& . \mathrm{I}_{\text {nom }}=43.3 \mathrm{kA} \text { with } \mathrm{B}_{\max }=4.5 \mathrm{~T}, \mathrm{~T} \sim 4.5 \mathrm{~K} . \\
& . \mathrm{I}_{\text {crit }}=125 \mathrm{kA} \text { for } \mathrm{B}_{\max }=5 \mathrm{~T}, \mathrm{~T}=4.2 \mathrm{~K} .
\end{aligned}
$$

The critical currents of both conductors were deduced from industrial production of the CMS conductor.

Only a small development is foreseen for the production of the larger cable, the smaller cable and the realization process being similar to the CMS ones.

\subsection{Winding}

The winding will be done using a similar multi layer inner winding technique already used for CMS [4]. In this technique, cylinders are used as external mandrels, the conductor being bent them pushed against them.

These external cylinders are very important pieces for the cold mass. They have four functions :

- external mandrel for the winding ;

- mechanical support for maintaining the electromagnetic bursting forces ;

- support of the cooling tubes which are welded on them, providing the indirect cooling of the coil by conduction through the cylinders ;

- quench back tube during a fast discharge.

They must have high mechanical properties. Al-alloy material will be used in this respect.

It is foreseen to have the winding of the five modules done by industry within its premises. The final assembly is foreseen to be done on the laboratory's site.

\subsection{Coil mechanical structure}

The electromagnetic forces will be contained both by the local reinforcement of the conductor, made of Al-alloy strips, and by the outer mandrels, thick enough to act as a mechanical support.

The TESLA conductor reinforcement has been designed a little bit larger than the CMS one to limit the maximum stress in the Al-alloy (120 MPa for TESLA vs 145 MPa for CMS), and to limit the strain to $0.15 \%$. 


\subsection{Cooling}

The cold mass will be indirectly cooled by saturated liquid helium at $4.5 \mathrm{~K}$, circulating in a thermosiphon mode, as for Aleph and CMS solenoids.

The cryogenics loads must take into account two $20 \mathrm{kA}$ current leads for the main current and two times two $25 \mathrm{kA}$ current leads for the correction current. The balance of the cryogenic loads is given in $\S 5.1$.

Cooling down times are foreseen to be :

- about one month from room temperature to $4.5 \mathrm{~K}$;

- about three days after a quench (in this case, the mean temperature of the coil is around $60 \mathrm{~K}$ ).

\subsection{Yoke}

Both the barrel and the end cap yokes will be built using iron plates, the dimensions of which have been taking into account production and transportation requirements.

The yoke assembly will not used welds, but bolts throughout, so that a trial assembly can be made before the final assembly.

The magnetic forces were calculated for the pole tips; the values are in the range of few MN on each disk.

The way to mechanically contain the forces on the end cap yokes in the long barrel version has to be studied in details.

\subsection{Assembly}

After the modules have been assembled, the cold mass will be completed with the thermal shields and the whole will be enclosed in the vacuum tank, which is the outer part of the cryostat.

The inner part of the vacuum tank will support the complete inner detector, including the mechanic and electromagnetic calorimeter and the TPC. Rails will be used.

The outer part of the vacuum tank will be supported in a cantilevered way by the central part of the barrel yoke.

The other parts of the barrel and end cap yoke will be assembled then, as four corner half shells. These parts must be easily movable for opening the magnet and accessing the various detector parts. Air pads are foreseen in this respect for axial and radial motions. 
A view of the cold mass with the yoke in the open position is given on Fig 4. More details about the mechanical assembly can be found in [5].

\subsection{Field mapping}

One of the big technological challenge of the magnet construction will be to actually ensure the requested field homogeneity. It implies that the $B_{x}$ and $B_{y}$ components have to be determined with a relative accuracy in the range of $0.2 \%$. A device with Hall probes, an updated version of those previously used for the mapping of the Aleph, Delphi an H1 magnets, can meet these requirements.

\section{5 - ANCILLARIES}

\subsection{Cryogenics plant}

The performance requirements of the cryogenic plant are deduced from the various cryogenic loads, which have been estimated to :

- $1 \mathrm{~kW}$ isothermal refrigeration at $4.5 \mathrm{~K}$;

- $4 \mathrm{~kW}$ non-isothermal refrigeration between $60 \mathrm{~K}$ and $80 \mathrm{~K}$ by helium gas ;

- liquid helium at a rate of $14 \mathrm{~g} / \mathrm{s}$ for current leads cooling between $4.5 \mathrm{~K}$ and $300 \mathrm{~K}$.

Moreover, this cryogenic plant must fulfill the time requirements for having the magnet in operation conditions as mentioned in $\S 4.4$.

This cryogenic plant could also be used, with some adaptation, for supplying the quadrupole doublets, foreseen to be working at $1.8 \mathrm{~K}(30 \mathrm{~W}$ at $1.8 \mathrm{~K}$ heat load for the two doublets).

The magnet cryogenic plant will include compressors, helium gas and liquid nitrogen storage containers, a cold box and the transfer lines. An intermediate cryostat for liquid helium storage (5 000 to 60001 capacity) will be also provided in case of failure of the main refrigeration system.

\subsection{Power circuit}

The electrical power circuit will consist of :

- a main bipolar power supply $(20 \mathrm{kA}, \pm 25 \mathrm{~V})$;

- two correction bipolar power supplies $(25 \mathrm{kA} \pm 5 \mathrm{~V})$, one for each correction winding ;

- the related switch breakers, dump resistances and electrical busbars for discharge of the magnet. 


\subsection{Control and monitoring system}

A conventional control and monitoring system will control and monitor the cryogenics and the electrical circuits and will provide the safety of the magnet.

\section{6 - ESTIMATES}

\subsection{Costs}

The cost estimate is based on industrial offers for the CMS magnet. A breakdown of the main components is given in Table IV. The total cost of the magnet adds up to $65 \mathrm{M}$ EUR with the following assumptions :

- the manpower costs listed are those for external manpower only (cost of manpower from laboratories is not included);

- there is no contingency.

$\underline{\text { Table IV : Cost estimate for the detector magnet }}$
Item
Cost (M EUR)

Coil

Conductor

Winding operation

10.0

Internal cryogenics and suspension

3.0

Tooling for assembly

1.2

Total for coil

Yoke and vacuum tank

Total for yoke and vacuum tank

Ancillaries

Cryogenic plant

Electrical power circuit

Control/monitoring system

1.3

Total for ancillaries

Miscellaneous (external manpower, test ...)

Total for miscellaneous 


\subsection{Manpower requirements}

As there is a lot of similarities between the TESLA and CMS magnets, few new developments are foreseen for the TESLA magnet, which will benefit from all the experience gained during the construction of the CMS magnet. Nevertheless, laboratory manpower will be needed for :

- the final design of the project (magnetic and mechanical calculations, drawing office) ;

- the following of industrial realizations (mainly conductor, winding and yoke);

- the scenario and following of the final assembly ;

- the tests.

It is estimated that a total of about 200 man.years are needed over the construction period of six years.

\subsection{Planning}

After approval of the project, a minimum period of six years is needed to build and test the magnet :

- one year to finalize the detailed design ;

- one year to place the main orders ;

- three year to produce the conductor, the modules and the yoke ;

- one year to do the final assembly and the cryogenic tests.

\section{7 - CONCLUSIONS}

The design of the TESLA detector magnet has already reached a mature state due to the fact that the magnet is similar to that of the CMS magnet. No major technical problems are foreseen, although both the absolute magnetic field of 4T and the homogeneity requirements are a challenge to the builders of this device. 


\section{REFERENCES}

[1] CMS collaboration, the Magnet Project, Technical Design Report, CERN, LHCC 97-10, May 1997.

[2] Proceeding of the Magnet Project Engineering Design Review, CERN, CMS 1999148, December 1998.

[3] I.L. Horwath at al., The CMS Conductor, IEEE Trans. on Applied Superconductivity, vol 10, no 1, 395, March 2000.

[4] P.F. Fabbricatore et al., Pre-industrialization Activities related to CMS Coil Winding, submitted to Applied Superconductivity Conference, Virginia Beach, USA, September 2000.

[5] N. Meyners, Mechanical Concept of the TESLA Detector, LC-DET-2001-045, DESY. 


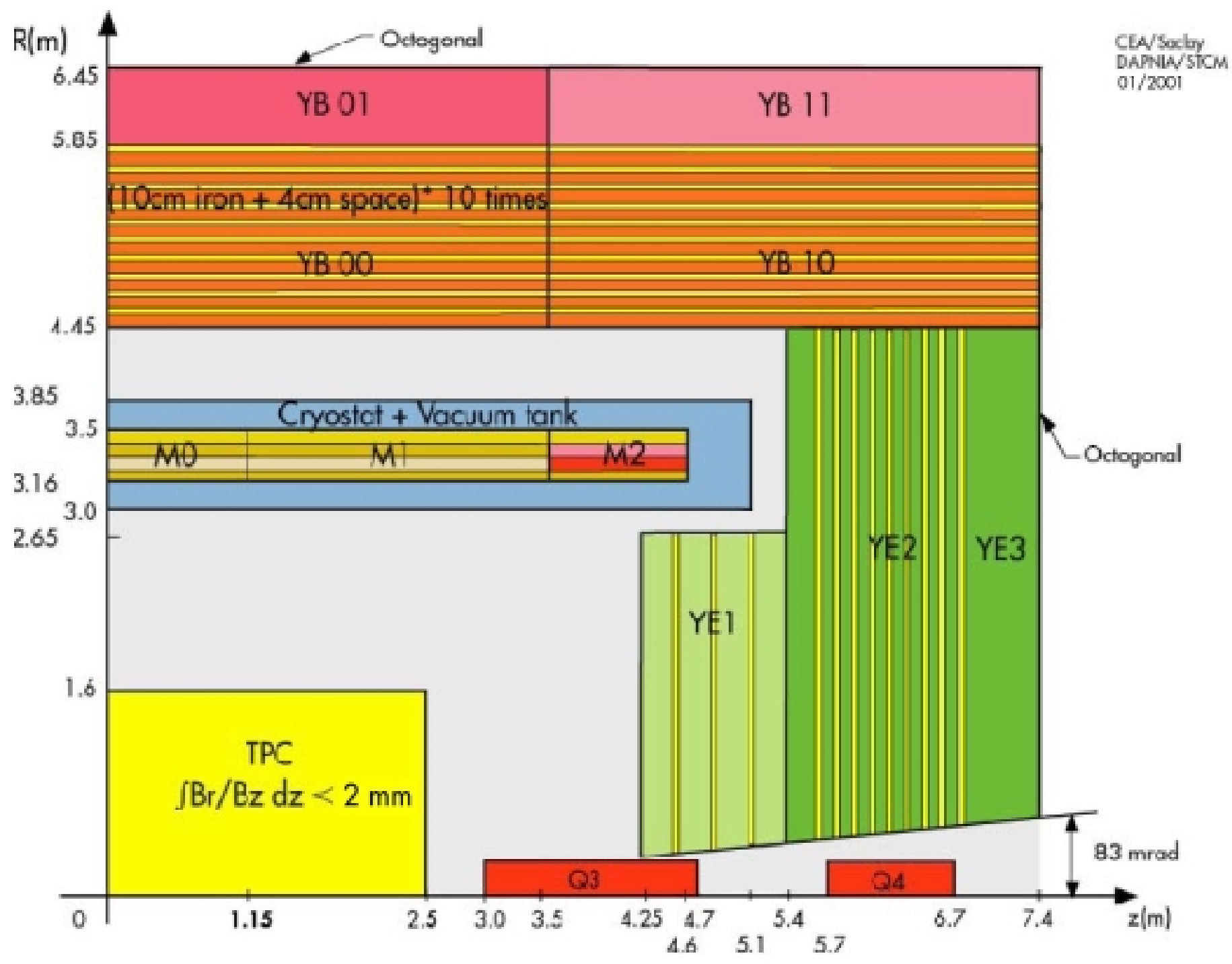

FIGURE 1. One quadrant of the TESLA detector magnet 


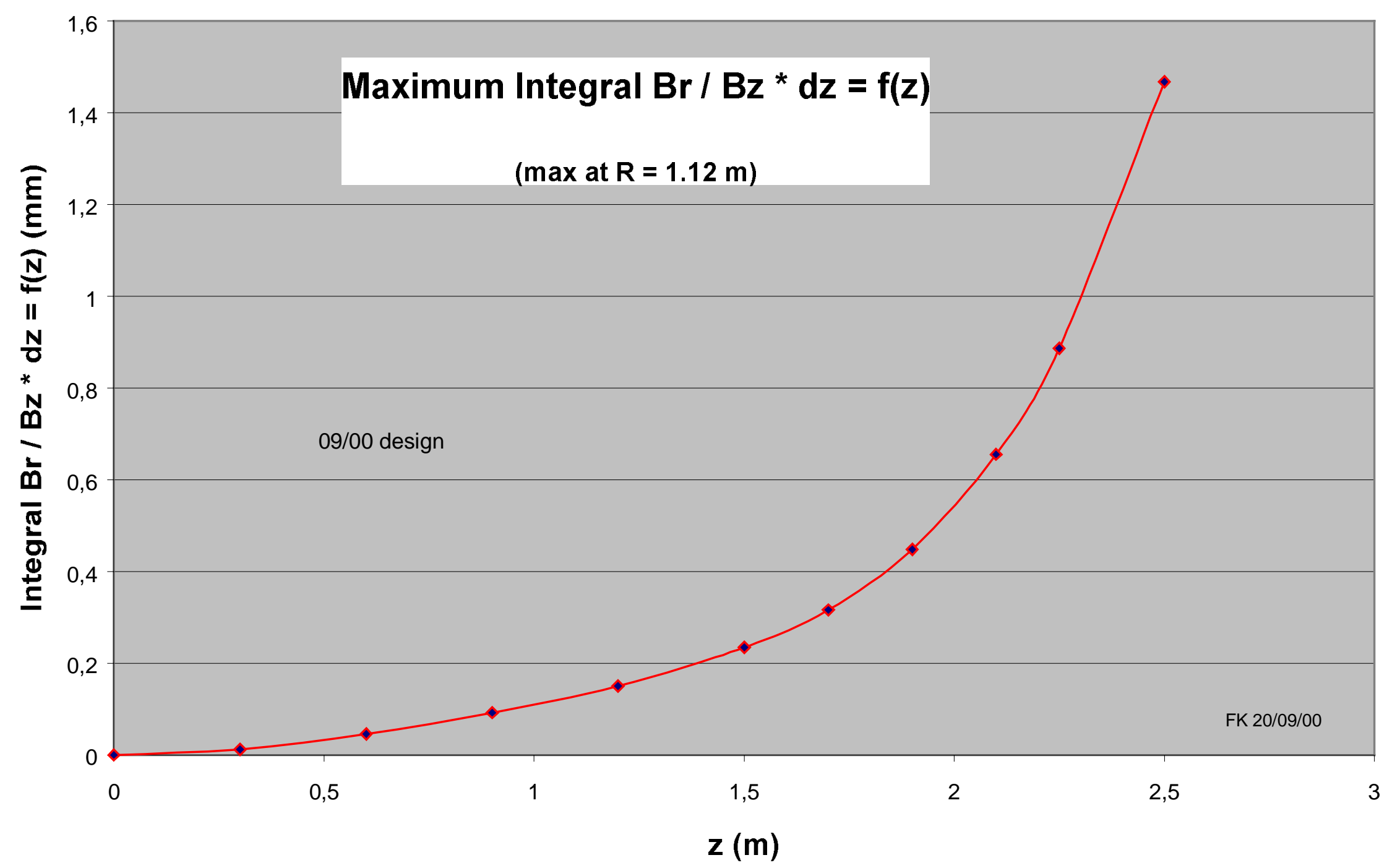

FIGURE 2. Maximum integrated field homogeneity 


\section{TESLA SC Conductor design}

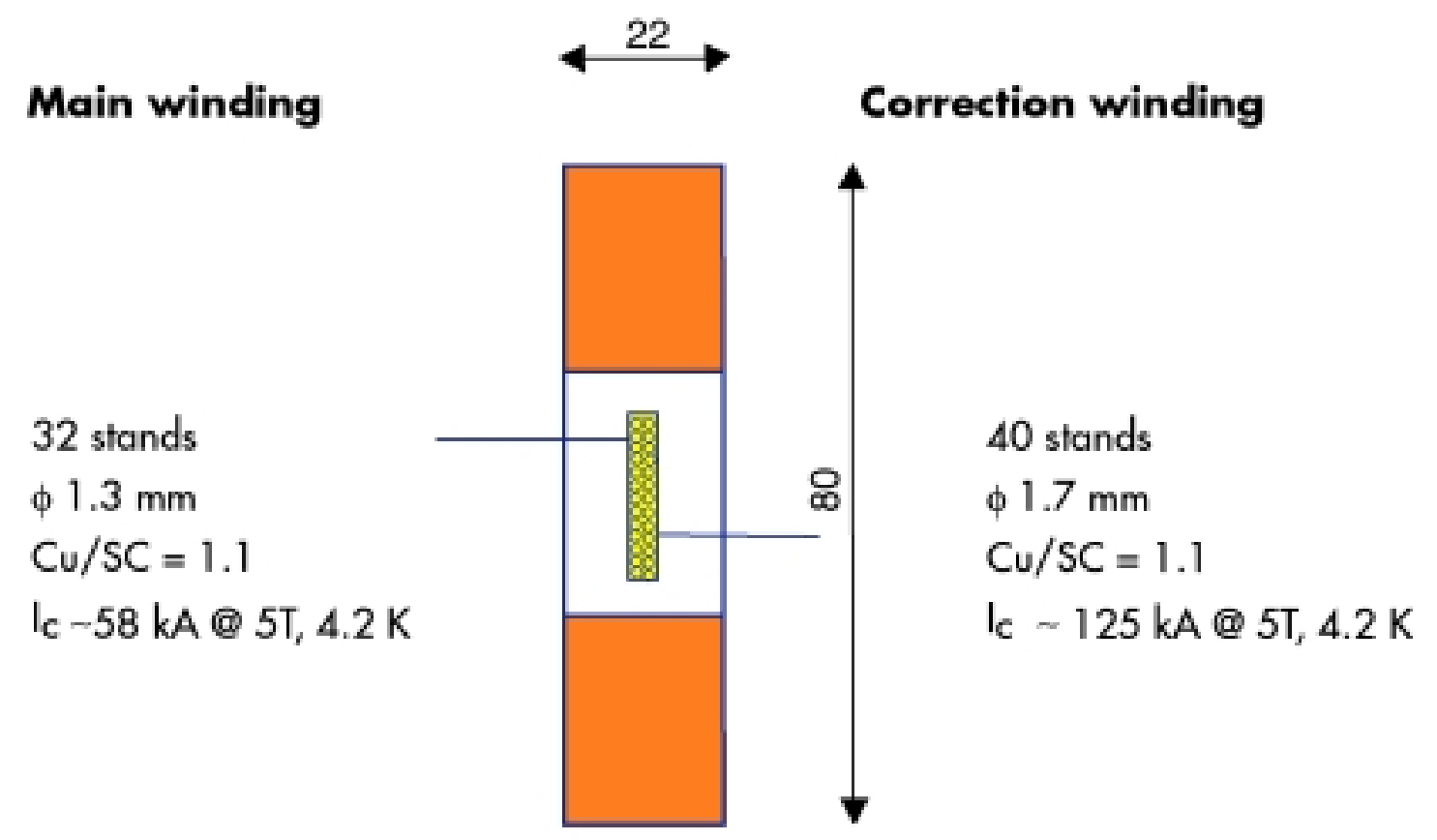

SC cable

High purity Al

Al alloy

CEA/Saclay

DAPNIA/STCM

$10 / 00$

FIGURE 3. TESLA conductor 


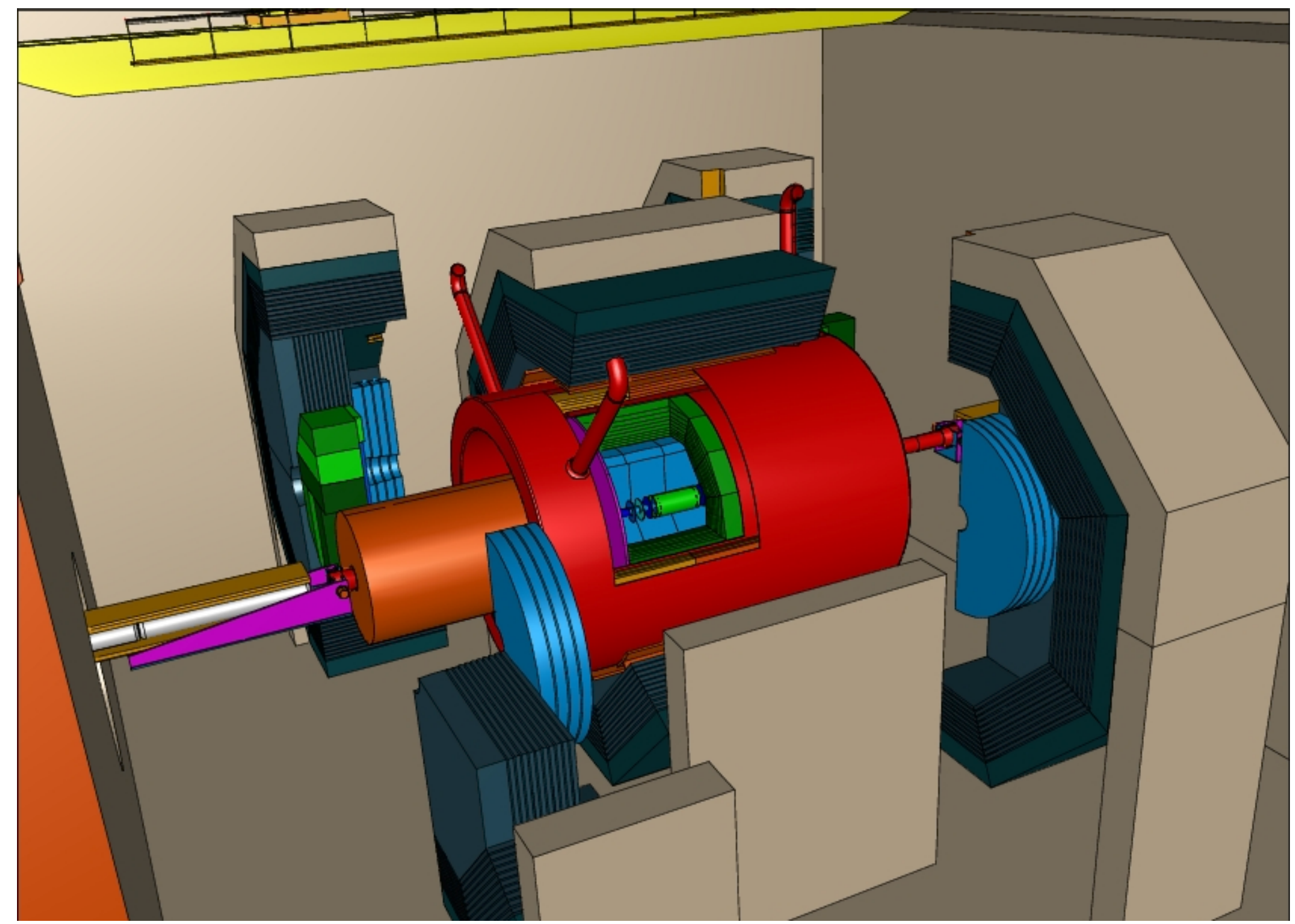

FIGURE 4 :The cold mass with the yoke in the open position 